\title{
Coordinating Independent Distributed Generators
}

\author{
J. A. Barros, Student Member, IEEE, H. Leite and N. Jenkins, Fellow, IEEE
}

\begin{abstract}
Developers of distributed generator schemes often wish to increase the size of their generators, in order to make their projects more economically attractive. This work reported the coordination of the operation of independent distributed generators connected to the same circuit, so as to maximise the amount of electrical energy they deliver without exceeding the power limit of that circuit. An Aggregator, owned by the generators, schedules the generators that can be dispatched in times of reduced output of the other generation. The coordination leads to an additional income from the surplus of electrical energy that is able to be produced. The Aggregator runs a cooperative game with transferable utility to allocate this amount, considering the electrical power produced and the type of each generator. The results of a numerical example showed that all generators have an economic incentive to operate in a coordinated manner.

Index Terms-- Cooperative game theory, coordinated operation, distribution networks, independent distributed generators, income sharing.
\end{abstract}

\section{INTRODUCTION}

$\mathrm{T}$ HE capacity of Distributed Generation continues to increase in many countries as efforts are made to decarbonise the power sector. Permission to connect a generator to the distribution system is generally obtained on the basis that the generator's effect is limited and that the network voltages and currents remain acceptable at all times. This fit-and-forget approach to connection limits the capacity of generation that can be connected [1].

A number of approaches to allow the connection of increased capacity of Distributed Generation have been suggested. Voltage control to mitigate the variations caused by Distributed Generation was presented in [2]. The strategic value of storage was assessed in [3]. Non-firm access to the network which allows the connection of capacities above the amount defined by the fit-and-forget approach was proposed in [4]. The Virtual Power Plant as a technical and commercial framework to aggregate a large quantity of distributed energy resources as a single operating profile was discussed in [5].

This work has been supported by the Fundação para a Ciência e Tecnologia (FCT) of Portugal, by means of the SFRH/BD/43688/2008 studentship.

J. A. Barros is with FE/UP (University of Porto, Faculty of Engineering), Porto, Portugal (contact author, e-mail: jose.barros@fe.up.pt)

H. Leite is with INESC Tec / FE/UP (University of Porto, Faculty of

Engineering), Porto, Portugal (e-mail: hleite@fe.up.pt)

N. Jenkins is with the with Institute of Energy, School of Engineering, Cardiff University, Cardiff, U.K. (e-mail: jenkinsn6@ cardiff.ac.uk).
Coordination of the operation of generators owned by one large producer [6] or by a number of smaller independent producers [7-9] has also been investigated. The business arrangements reported in $[8,9]$ to reward independent producers that coordinate their output are based on Cooperative Game Theory. When agents form a coalition to maximise a common benefit, Cooperative Game Theory allows them to negotiate binding and enforceable agreements to allocate that common benefit $[10,11]$.

Some Distributed Generation can be dispatched (e.g., some forms of Combined Heat and Power) while other technologies (e.g., wind and solar) respond to environmental conditions. This paper addresses the coordination of the operation of dispatchable and non-dispatchable distributed generators owned by independent energy producers. When non-firm access to the network is allowed, the coordination allows generators to produce additional amounts of electrical energy without exceeding the power capacity limit of the network. The additional income obtained when operating in a coordinated manner is allocated amongst all generators using a cooperative game with transferable utility [12]. An Aggregator, run by the generators, schedules the generators to produce additional electrical energy and runs the cooperative game to allocate the resultant additional income.

\section{COORDINATED OPERATION OF INDEPENDENT DISTRIBUTED GENERATORS}

Operators of distribution networks limit the connection of generation to their circuits in order to ensure acceptable voltages and currents at all times. However, to make projects more economically attractive, developers of distributed generator schemes often wish to increase the size of their generators. The work reported here coordinates the operation of independent distributed generators connected to the same circuit so as to maximise the amount of electrical energy they deliver without exceeding the power limit of that circuit. Those generators that can be dispatched are asked to change their generation output to times of reduced output of the other generation. However, if all the generators receive their income directly from the energy they produce, there would be no economic incentive for some of them to act in a coordinated manner. Therefore, all generators receive an allocation of the additional income set by a cooperative game with transferable utility. In this allocation, the amount of electrical energy produced and the type of each generator are considered. 


\section{A. Description of the Coordination of Independent Distributed Generators}

The coordination of independent distributed generators is described using a general example. Consider three independent distributed generators (GenA, GenB and GenC) connected to the same distribution circuit, with no local loads. GenA and GenC are non-dispatchable generators (e.g., wind generators), while GenB is a dispatchable generator (e.g., a thermal generator). GenA and GenB were connected first to the circuit and granted firm access (i.e., they have the right to export up to their rating at all times). The sum of the installed capacities of GenA and GenB equals the power rating of the circuit (Pcircuit). GenC was connected to the circuit later and agreed to operate on the basis of non-firm access (i.e. to be constrained off when there was inadequate capacity in the export circuit). Therefore, two scenarios may occur in the operation of these generators:

I. The output powers of the generators do not exceed the circuit rating;

II. The output powers of the generators exceed the circuit rating and GenC is curtailed to meet the circuit rating limit [7, $9,13]$.

In this work a third scenario for the operation of the generators is introduced:

III. The output powers of the generators exceed the circuit rating but they agree to be operated in a coordinated manner so as to maximise the electrical energy produced without exceeding the circuit rating.

The coordination of generators is operated by an Aggregator, run by the generators. The Aggregator schedules the dispatchable generator GenB to allow the non-dispatchable generator GenC to operate. GenB is scheduled to operate in the hours of reduced output of the non-dispatchable generators. GenC is curtailed when the circuit rating limit is still exceeded. GenA is not curtailed because it is a nondispatchable generator with firm access.

The Aggregator only operates when the following set of conditions is met:

$a$. The output powers of the generators exceed the circuit rating;

$b$. GenB does not need to operate at full output power during the whole period of operation.

If condition $a$. is not met, all generators produce according to their projections (scenario I.). If condition $b$. is not met, GenC is curtailed to meet the circuit rating limit (scenario II.).

The Aggregator uses the following parameters of the system for its operation: circuit rating (considered to be fixed); operating limits of GenB and GenC; fuel and start-up costs of GenB.

The operation of the Aggregator is as follows:

(1) The Aggregator receives from the generators their anticipated outputs and the amount of time that GenB can produce continuously at full output power. GenA and GenC make their projections based on power forecasts. GenB makes its projection considering the prediction of the price of electrical energy and any need to produce heat;
(2) The Aggregator assesses whether the anticipated outputs exceed the circuit rating. If the limit is not exceeded, the Aggregator accepts the anticipated outputs and takes no further action. If the limit is exceeded, the Aggregator runs an optimisation algorithm to schedule GenB and GenC.

(3) The optimisation algorithm has an objective function ((1)) to maximise the income of the generators (variable $I$ ).

$$
\max I=\sum_{h=1}^{H}\left(\rho_{h} \cdot u_{h} \cdot P B_{h}-\text { Fuel }_{h}-\text { Start }_{h}\right)+\sum_{h=1}^{H}\left(\rho_{h} \cdot P C_{h}\right.
$$

The first term of Equation (1) is the income of GenB and the second term is the income of GenC. $h$ is the time step of the optimisation; $H$ is the time span of the optimisation; $\rho$ is the price of electrical energy; $u$ is a binary variable that indicates whether GenB is operating; $P B$ and $P C$ are the output powers of GenB and GenC, respectively; Fuel and Start are the terms for the production and start-up costs of GenB and are given by (2) and (3), respectively [14]:

$$
\begin{gathered}
\text { Fuel }_{h}=f c \cdot u_{h} \cdot P B_{h} \\
\text { Start }_{h}=\max \left(0, s u \cdot\left(u_{h}-u_{h-1}\right)\right)
\end{gathered}
$$

$f c$ and $s u$ are the fuel and start-up costs of GenB. The production costs of GenC are not considered. The costs of power forecasting are not considered. The costs with the prediction of the price of electrical energy are also not considered in the objective function.

The constraints of the optimisation algorithm are the power limit of the distribution circuit ((4)), the operating limits of GenB and GenC ((5) and (6)) and the amount of electrical energy that GenB can produce ((7)).

Power limit of the distribution circuit

$$
\begin{aligned}
& P A \_a n t_{h}+P B_{h}+P C_{h} \leq P \text { circuit, } \forall h \in\{1, \ldots, H\} \\
& \text { Operating limits of the generators } \\
& \text { Generator GenB } \\
& \quad P B_{\min } \leq P B_{h} \leq P B_{\max }, \forall h \in\{1, \ldots, H\} \\
& \text { Generator GenC } \\
& \quad P C_{\min } \leq P C_{h} \leq P C C_{-} \text {ant } t_{h}, \forall h \in\{1, \ldots, H\} \\
& \text { Amount of electrical energy that GenB can produce } \\
& \sum_{h=1}^{H}\left(u_{h} \cdot P B_{h}\right)-\left(\text { TFull } \cdot P B_{\max }\right) \leq 0
\end{aligned}
$$

PA_ant is the anticipated output of GenA; PBmin and $P C \min$ are the minimum output powers of GenB and GenC, respectively; PBmax is the rated power of GenB; PC_ant is the anticipated power of GenC; TFull is the amount of time that GenB can produce continuously at full output power.

(4) The outcome of the optimisation is the coordinated output of the generators that maximises the electrical energy they produce and does not exceed the circuit rating.

The operation of the Aggregator is illustrated in Fig. 1.

\section{B. Allocating the Additional Income using Cooperative Game} Theory

The coordinated output of the generators leads to an additional income from the additional electrical energy that is able to be produced. This additional electrical energy is the result of changing the output of GenB to let GenC operate when 
otherwise it would be curtailed. The generators with firm access (GenA and GenB) would have no economic incentive to participate in the coordination if their income came directly from the electrical energy they produced. Thus, the Aggregator allocates the additional income using a cooperative game with transferable utility.

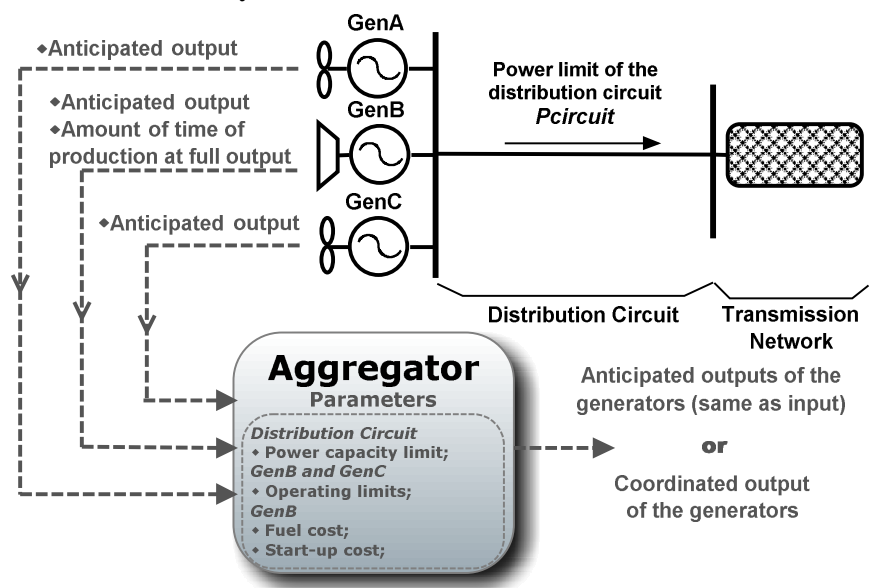

Fig. 1. Distribution circuit with three independent distributed generators and the Aggregator.

Before the allocation, the Aggregator assesses whether any generator did not comply with its coordinated output (or anticipated output, in the case of GenA). If so, that generator is penalised for the deviation. The allocation is made as follows:

(i) The additional income is obtained using (8). Equation (8) is the difference between the income received in coordination and the income received if GenC were curtailed. This value is the worth of the coalition of all generators.

$$
\begin{aligned}
& \delta=\left(\sum_{h=1}^{H}\left(\rho_{h} \cdot u_{h} \cdot P B_{h}-\text { Fuel }_{h}-\text { Start }_{h}\right)+\sum_{h=1}^{H}\left(\rho_{h} \cdot P C_{h}\right)\right)- \\
& \left(\sum_{h=1}^{H}\left(\rho_{h} \cdot P B \_a n t_{h}-\text { Fuel_ant }_{h}-\text { Start_ant }_{h}\right)+\sum_{h=1}^{H}\left(\rho_{h} \cdot P C_{-} \text {Curt }_{h}\right)\right)
\end{aligned}
$$

PB_ant is the anticipated output of GenB; Fuel_ant and Start_ant are the terms for the production and start-up costs of GenB in its projected operation; PC_curt is the curtailed output of GenC.

(ii) The worth of the coalition is allocated using a concept of Cooperative Game Theory called "the core" [15]. The core of this cooperative game is the set of allocations that meet three criteria: (1) the additional income is entirely allocated; (2) all generators receive a greater income in coordination (when compared with the condition when GenC is curtailed); (3) the coalition of all generators provides a greater income for any generator when compared to the income any generator would receive in a coalition of two generators. Any allocation of the core provides all generators the economic incentive to operate in a coordinated manner. This cooperative game is a convex game, given that the worth of the coalition increases as the coalition grows. Therefore, the core of this cooperative game is always non-empty [15]. The core of this cooperative game can be a large set of allocations, each of them giving different economic incentives to the generators, as illustrated by the hatched area of Fig. 2.

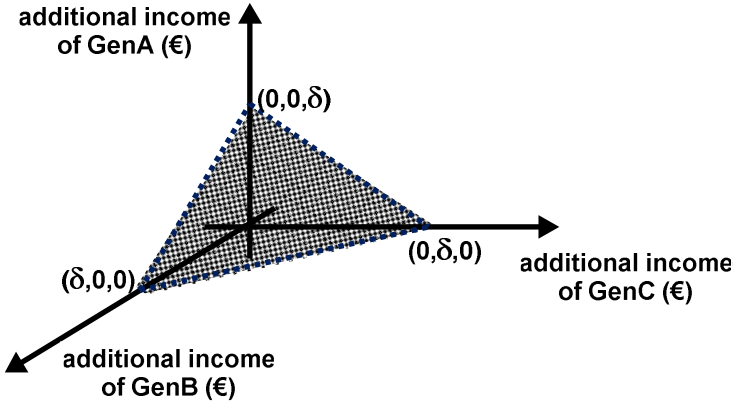

Fig. 2. Core of this cooperative game (hatched area).

So, the Aggregator chooses a single allocation from the core (allocA, allocB, alloc C) using (9). This choice is made by weighting the amount of electrical energy produced and the type of each generator. The type of generation is differentiated using dimensionless coefficients (coefA, coefB and coefC), agreed beforehand by the generators. The coefficients are likely to be based on the subsidies received for each technology type.

$$
\left\{\begin{array}{l}
\text { allocA }=\frac{\text { coefA } \cdot \sum_{h=1}^{H} P A_{-} \text {ant }_{h}}{\operatorname{coefA} \cdot \sum_{h=1}^{H} P A_{-} \text {ant } t_{h}+\operatorname{coefB} \cdot \sum_{h=1}^{H}\left(u_{h} \cdot P B_{h}\right)+\operatorname{coefC} \cdot \sum_{h=1}^{H} P C_{h}} \\
\text { allocB }=\frac{\operatorname{coefB} \cdot \sum_{h=1}^{H}\left(u_{h} \cdot P B_{h}\right)}{\operatorname{coefA} \cdot \sum_{h=1}^{H} P A_{-} \text {ant } t_{h}+\operatorname{coefB} \cdot \sum_{h=1}^{H}\left(u_{h} \cdot P B_{h}\right)+\operatorname{coefC} \cdot \sum_{h=1}^{H} P C_{h}} \\
\text { allocC }=\frac{\operatorname{coefC} \cdot \sum_{h=1}^{H} P C_{h}}{\operatorname{coefA} \cdot \sum_{h=1}^{H} P A_{-} \_a n t_{h}+\operatorname{coefB} \cdot \sum_{h=1}^{H}\left(u_{h} \cdot P B_{h}\right)+\operatorname{coefC} \cdot \sum_{h=1}^{H} P C_{h}}
\end{array}\right.
$$

(iii) Equation (10) gives the income that each generator receives in coordination (income $\mathrm{A}$, income $\mathrm{B}$ and incomeC). The allocation to each generator is added to the income it would have received if GenC were curtailed (i.e., without coordination).

$$
\left\{\begin{array}{l}
\text { income } A=\sum_{h=1}^{H}\left(\rho_{h} \cdot P A \_a n t_{h}\right)+(\text { allocA } \cdot \delta) \\
\text { income } B^{H}=\sum_{h=1}^{H}\left(\rho_{h} \cdot P B \_ \text {ant }_{h}-\text { Fuel_ant }_{h}-\text { Start_ant }_{h}\right)+\left(\text { allocB }^{\prime} \delta\right) \\
\text { income } C=\sum_{h=1}^{H}\left(\rho_{h} \cdot P C \_ \text {curt }_{h}\right)+(\text { alloc } C \cdot \delta)
\end{array}\right.
$$

Note that GenA receives a greater income despite not being scheduled. This is because GenB cannot change its output without knowing the anticipated output of GenA. Therefore, GenA is rewarded for communicating its anticipated output to the Aggregator and covering the risk of being penalised for not complying with it.

\section{NUMERICAL EXAMPLE}

A simple numerical example over 24 hours illustrates the process. The set of conditions for the operation of the Aggregator is met. GenA and GenC are wind generators. GenB is a biomass generator. Parameters of the system are given in Table I. 
TABLE I

PARAMETERS OF THE SYSTEM

\begin{tabular}{l|c|c}
\hline Parameter & Value & Unit \\
\hline Power capacity limit of the distribution & 26.50 & MW \\
circuit $($ Pcircuit $)$ & {$[3.62 ; 9.05]$} & MW \\
Operating limits of GenB $\left(\left[P B_{\min } ; P B_{\max }\right]\right)$ & 0 & MW \\
Minimum output power of GenC $\left(P C_{\min }\right)$ & 35 & $(€ / \mathrm{MWh})$ \\
Fuel cost of GenB $(f c)$ & 100 & $(€)$ \\
Start-up cost of GenB $(s u)$ & &
\end{tabular}

The price of electrical energy is shown in Fig. 3.

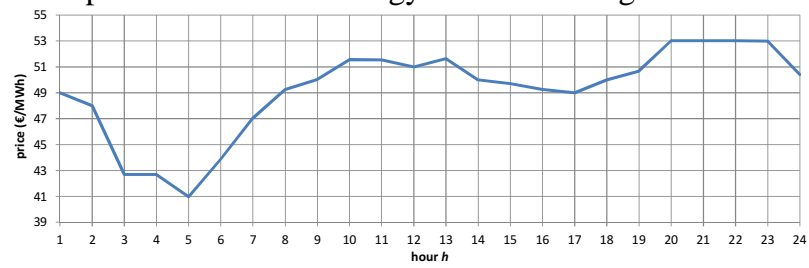

Fig. 3. Price of electrical energy (Iberian daily market - Portugal 14/03/2011. Source: http://www.omie.es/en/inicio).

\section{A. Generators communicate their anticipated output to the Aggregator}

The generators communicate their anticipated outputs to the Aggregator, as illustrated in Figs. 4 to 6. GenA and GenC follow the wind power forecasts. GenB predicts its output following the price profile given in Fig. 3. GenB operates at full output for 16.7 hours.

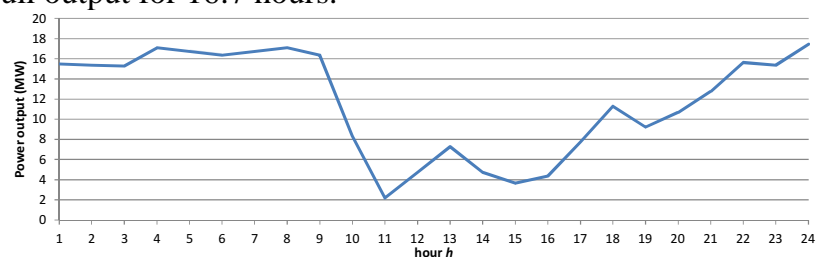

Fig. 4. Anticipated output of GenA.

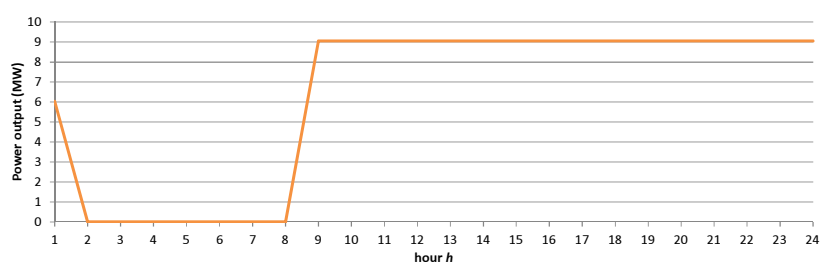

Fig. 5. Anticipated output of GenB.

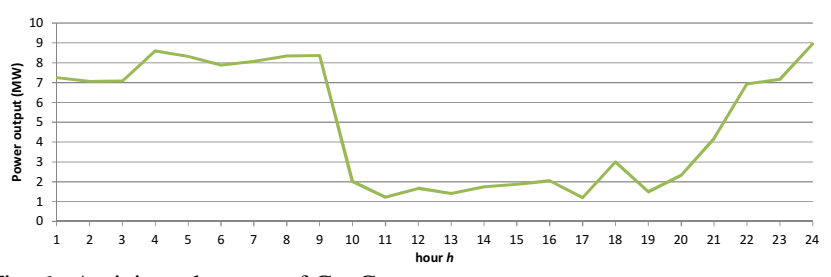

Fig. 6. Anticipated output of GenC.

Fig. 7 shows the sum of the anticipated outputs versus the circuit rating.

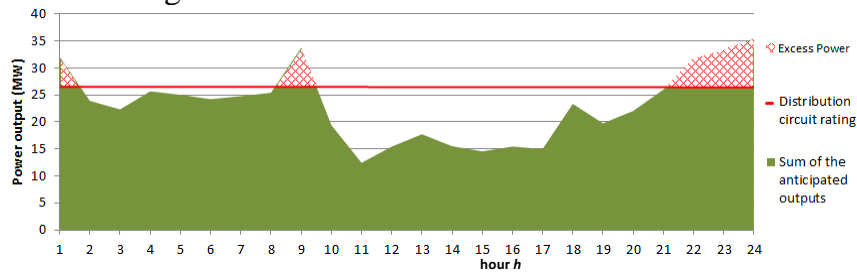

Fig. 7. Sum of the anticipated outputs.
The rating is exceeded in hours $1,9,22,23$ and 24 by the sum of the anticipated outputs.

\section{B. Power Curtailment to meet the Power Limit of the Distribution Circuit}

The electrical energy produced and the income of each generator if $\mathrm{GenC}$ were curtailed to meet the circuit limit are given in Table II. GenB is considered to be operating before hour 1.

TABLE II

ELECTRICAL ENERGY PRODUCED AND INCOME RECEIVED BY EACH GENERATOR IF GENC WERE CURTAILED

\begin{tabular}{c|c|c|c}
\hline & GenA & GenB & GenC \\
\hline Electrical energy produced $(\mathrm{MWh})$ & 281.93 & 150.83 & 86.51 \\
\hline Income received $(€)$ & 13700 & 2309 & 4080 \\
\hline
\end{tabular}

\section{Generators in Coordinated Operation}

Coordinated operation of the generators maximises the energy they produce without exceeding the circuit rating. The Aggregator schedules the output power of GenB to avoid curtailing GenC. The coordinated output of GenB is shown in Fig. 8.

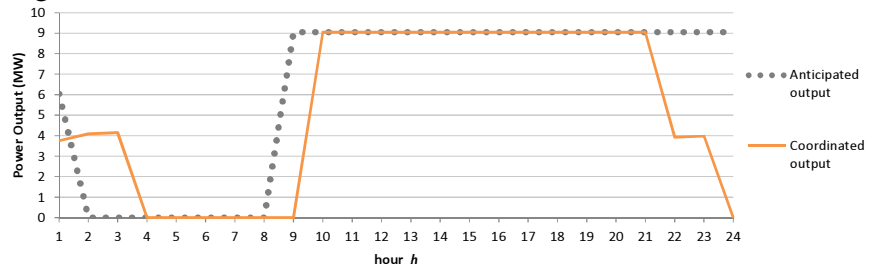

Fig. 8. Coordinated output of GenB.

The coordinated output of GenB and GenC provides an income of $7598 €$.

\section{Allocating the Additional Income obtained in Coordinated Operation}

If the income received by each generator came directly from the electrical energy produced, GenA would receive $13700 €$ (as it maintained its anticipated output), GenB would receive $1903 €$ and GenC would receive $5694 €$. GenA and GenB would have no economic incentive to be operated in a coordinated manner, preferring that GenC is curtailed. If the generators operate in a coordinated manner, the additional income is:

$$
\delta=7598-(2309+4080)=1209 € .
$$

The allocation of the additional income chosen by the Aggregator is given in Table III. In this example, the Aggregator uses the coefficients for each generation type set by the Portuguese Government. In Portugal, the formula established by legislation to remunerate renewable generators contains a coefficient that differentiates the type of generation. 4.6 is the coefficient set for onshore wind generators (GenA and GenC) and 8.2 is the coefficient set for non-CHP biomass generators based on forestry residues (GenB) [16]. 
TABLE III

ALLOCATION OF THE ADDITIONAL INCOME

\begin{tabular}{c|c|c|c}
\hline & GenA & GenB & GenC \\
\hline Electrical energy produced (MWh) & 281.93 & 128.49 & 118.22 \\
\hline $\begin{array}{c}\text { Coefficient for generation } \\
\text { type(dimensionless) }\end{array}$ & 4.6 & 8.2 & 4.6 \\
\hline Allocation (dimensionless) & $\mathbf{0 . 4 5}$ & $\mathbf{0 . 3 6}$ & $\mathbf{0 . 1 9}$ \\
\hline Amount of additional income (€) & $\mathbf{5 4 2}$ & $\mathbf{4 4 0}$ & $\mathbf{2 2 7}$ \\
\hline
\end{tabular}

The allocation of the additional income chosen by the Aggregator is shown in Fig. 9.

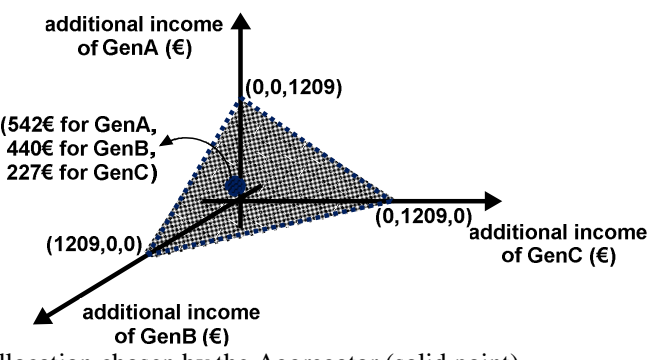

Fig. 9. Allocation chosen by the Aggregator (solid point).

Table IV shows a comparison of the income received by each generator.

TABLE IV

COMPARISON OF THE INCOME RECEIVED BY EACH GENERATOR

\begin{tabular}{c|c|c|c|c}
\hline & GenA & GenB & GenC & TOTAL \\
\hline GenC curtailed $(€)$ & 13700 & 2309 & 4080 & $\mathbf{2 0 0 8 9}$ \\
\hline $\begin{array}{c}\text { Coordinated } \\
\text { operation }(€)\end{array}$ & 14242 & 2749 & 4307 & $\mathbf{2 1 2 9 8}$ \\
\hline Variation & $\mathbf{+ 3 . 9 6 \%}$ & $\mathbf{+ 1 9 . 0 6 \%}$ & $\mathbf{+ 5 . 5 6 \%}$ & $\mathbf{+ 6 . 0 2} \%$ \\
\hline
\end{tabular}

\section{CONCLUSION}

Coordination of independent distributed generators maximises the energy they produce without exceeding the distribution circuit rating. An Aggregator, run by the generators, schedules the dispatchable generators to allow the non-dispatchable generators to operate. The dispatchable generators are scheduled in hours of reduced output of the non-dispatchable generators. The coordinated operation of the generators leads to an additional income from the additional electrical energy that is able to be produced. The Aggregator runs a cooperative game with transferable utility to allocate this additional income.

In the numerical example shown, GenB is scheduled to allow GenC to operate. The increase in the production of electrical energy of GenC and the scheduling of GenB in hours of reduced output of the wind generators leads to an additional income. It was shown that all generators increase their income, having an economic incentive to act in a coordinated manner.

\section{REFERENCES}

[1] T. Boehme, G. P. Harrison, and A. R. Wallace, "Assessment of distribution network limits for non-firm connection of renewable generation," IET Renewable Power Generation, vol. 4, pp. 64-74, 2010.

[2] P. M. S. Carvalho, P. F. Correia, and L. A. F. Ferreira, "Distributed Reactive Power Generation Control for Voltage Rise Mitigation in Distribution Networks," Power Systems, IEEE Transactions on, vol. 23, pp. 766-772, 2008
[3] DOE-Electricity-Advisory-Committee, "Bottling Electricity: Storage as a Strategic Tool for Managing Variability and Capacity Concerns in the Modern Grid," 2008, pp. 1-40.

[4] S. N. Liew and G. Strbac, "Maximising penetration of wind generation in existing distribution networks," Generation, Transmission and Distribution, IEE Proceedings, vol. 149, pp. 256-262, 2002.

[5] "FENIX Project - Flexible Electricity Network to Integrate the expected "energy evolution"." vol. European Union Project, available online: http://fenix-project.org (last accessed on 29/03/2011), 2005-2009.

[6] J. M. Angarita and J. G. Usaola, "Combining hydro-generation and wind energy: Biddings and operation on electricity spot markets," Electric Power Systems Research, vol. 77, pp. 393-400, 2007.

[7] S. C. E. Jupe, P. C. Taylor, and A. Michiorri, "Coordinated output control of multiple distributed generation schemes," Renewable Power Generation, IET, vol. 4, pp. 283-297, 2010.

[8] N. X. Jia and R. Yokoyama, "Profit allocation of independent power producers based on cooperative Game theory," International Journal of Electrical Power \& Energy Systems, vol. 25, pp. 633-641, 2003.

[9] M. Zima-Bockarjova, J. Matevosyan, M. Zima, and L. Soder, "Sharing of Profit From Coordinated Operation Planning and Bidding of Hydro and Wind Power," Power Systems, IEEE Transactions on, vol. 25, pp. $1663-1673,2010$

[10] H. Singh, "Introduction to game theory and its application in electric power markets," IEEE Computer Applications in Power, vol. 12, pp. 18-20, 22, 1999.

[11] K. Shaloudegi, N. Madinehi, S. H. Hosseinian, and H. A. Abyaneh, "A Novel Policy for Locational Marginal Price Calculation in Distribution Systems Based on Loss Reduction Allocation Using Game Theory," Power Systems, IEEE Transactions on, pp. 1-10, 2012.

[12] H. Peters, Game Theory: A Multi-Leveled Approach: Springer, ISBN: 3540-69290-8, pp. 121-131, 2008.

[13] M. J. Dolan, E. M. Davidson, G. W. Ault, and J. R. McDonald, "Techniques for managing power flows in active distribution networks within thermal constraints," in CIRED 2009. 20th International Conference and Exhibition on Electricity Distribution - Part 1, , 2009, , pp. $1-4$.

[14] A. T. Al-Awami and M. A. El-Sharkawi, "Coordinated Trading of Wind and Thermal Energy," Sustainable Energy, IEEE Transactions on, vol. 2, pp. 277-287, 2011.

[15] L. S. Shapley, "Cores of convex games," International Journal of Game Theory, vol. 1, pp. 11-26, 1971.

[16] "National Renewable Energy Action Plan for Portugal under Directive 2009/28/CE," Goverment of the Portuguese Republic, available online: http://ec.europa.eu/energy/renewables/transparency_platform/action_p lan_en.htm (last accessed on 13/03/2012), Lisbon, June 2010.

\section{BIOGRAPHIES}

Jose Alberto Barros (StM'12) received his Graduate and MSc degrees from the Faculty of Engineering of the University of Porto (FE/UP), Portugal, in 2007 and 2008, respectively. Jose Alberto Barros is currently pursuing a PhD degree at the Department of Electrical and Computer Engineering of FE/UP, in collaboration with the School of Engineering at Cardiff University, U.K.

Helder Leite received his Electrical Engineering degree from the University of Porto, Portugal in 2000 and the $\mathrm{PhD}$ degree in Electrical Engineering from The University of Manchester, UK, in 2004. Helder Leite joined University of Porto, Portugal as a Lecturer in 2005. His research interests include Distribution Generation Integration, Electric Power Systems and Power Systems Protection.

Nick Jenkins (SM'97-F'05) received the Ph.D.degree from Imperial College, London, U.K., in 1986. He is the Director of the Institute of Energy within the School of Engineering at Cardiff University, U.K. Before moving to academia, his career included 14 years industrial experience, of which 5 years were in developing countries. His final position in industry was as Projects Director for Wind Energy Group, a manufacturer of large wind turbines. While at University he has developed teaching and research activities in both electrical power engineering and renewable energy.

Dr. Jenkins is a Fellow of the IET and the Royal Academy of Engineering and the Shimizu Visiting Professor to the Atmosphere and Energy Program at Stanford University. 